13

\title{
Эффективные широкополосные приемники терагерцового излучения на основе болометров с тонким металлическим поглотителем
}

\author{
(C) М.А. Демьяненко \\ Институт ффизики полупроводников им. А.В. Ржанова СО РАН, \\ 630090 Новосибирск, Россия \\ e-mail: demyanenko@isp.nsc.ru
}

(Поступило в Редакцию 11 января 2017 г. В окончательной редакции 12 апреля 2017 г.)

На основе матричного метода проведен расчет коэффициентов поглощения терагерцового излучения в традиционном и инвертированном типе болометрических структур, отличающихся тем, что в первом случае излучение падает со стороны прилегающего к болометру вакуума, а во втором - со стороны подложки, на которой он изготовлен. Показано, что применение дополнительного резонатора в виде зазора между болометром и входным или выходным окном позволяет получать коэффициенты поглощения близкие к единице, при этом болометры традиционного типа становятся узкополосными, а инвертированного остаются широкополосными.

DOI: 10.21883/JTF.2018.01.45495.2165

\section{Введение}

Последние полтора десятилетия проявляется значительный интерес к детектированию и визуализации излучения терагерцового спектрального диапазона $(0.3-10 \mathrm{THz})$ для создания систем безопасности, в биохимических, медицинских и других целях $[1,2]$. Системы визуализации с активной подсветкой на длине волны около $100 \mu \mathrm{m}$, работающие на кадровой частоте вплоть до $90 \mathrm{~Hz}$, были реализованы на основе матричных микроболометрических приемников и достаточно мощных газовых [3] и квантовых каскадных [4] лазеров, а также лазеров на свободных электронах [5]. Применяемые в этих работах стандартные микроболометры были разработаны для инфракрасного диапазона $8-14 \mu \mathrm{m}$, в котором они обладают высокой чувствительностью мощность, эквивалентная шуму (Noise Equivalent Power, $\mathrm{NEP}$ ), составляет около $1 \mathrm{pW} / \mathrm{Hz}^{1 / 2}$ [4]. Однако на длинах волн, близких к $100 \mu \mathrm{m}$, их чувствительность оказалась много ниже - NEP $=200-300 \mathrm{pW} / \mathrm{Hz}^{1 / 2}[4-5]$, что преимущественно обусловлено малой величиной пропускания используемых германиевых окон $(\sim 25 \%)$ и малым коэффициентом поглощения терагерцового излучения в микроболометрах $(\sim 3 \%)[6]$.

В дальнейшем величина коэффициента поглощения была значительно повышена путем нанесения дополнительного тонкого металлического поглотителя на поверхность микроболометра, что вместе с заменой германиевого окна на кремниевое, просветленное в рабочем диапазоне длин волн, и значительным понижением теплопроводности балок, поддерживающих болометр, позволило понизить NEP до $\sim 40 \mathrm{pW}$ [6]. Отметим, что размерность $\mathrm{pW}$ подразумевает мощность, эквивалентную шуму, в полосе частот интегрирования сигнала (для матричных приемников ее характерное значение равно $10 \mathrm{kHz}$ ), в то время как размерность $\mathrm{pW} / \mathrm{Hz}^{1 / 2}$ означает мощность, эквивалентную шуму, в полосе
$1 \mathrm{Hz.}$ Увеличение высоты подвески микроболометра над кремниевой подложкой, в которой выполнена схема считывания сигналов с 3.2 до $5.7 \mu \mathrm{m}$, позволило еще повысить коэффициент поглощения и понизить NEP в 1.4 раза [7]. Отметим, что все вышеперечисленные меры повышения чувствительности микроболометра в терагерцовом диапазоне позволяли сохранить широкополосность приемника.

Применение дополнительного оптического резонатора, образованного $50 \mu \mathrm{m}$ зазором между микроболометром и входным кремниевым окном, привело к дальнейшему понижению NEP до $\sim 17 \mathrm{pW}$ на длине волны, равной $97 \mu \mathrm{m}$ [7], однако при этом приемник стал узкополосным. Использование дополнительного интегрирования по пикселям и кадрам, что, естественно, приводит к понижению пространственного и временно́го разрешения, позволило понизить $\mathrm{NEP}$ до $\sim 1 \mathrm{pW}[6]$.

В настоящей работе проводится сравнительный анализ коэффициентов поглощения терагерцового излучения в болометрических структурах с тонким металлическим поглотителем традиционного и предлагаемого нами инвертированного типа с целью выявления конфигурации, позволяющей создать широкополосный терагерцовый болометрический приемник, обладающий коэффициентом поглощения, близким к единице.

\section{1. Предварительный анализ коэффициентов поглощения в болометрических приемниках традиционного и инвертированного типа}

Все вышеуказанные модификации дизайна болометра соответствуют традиционной конфигурации (рис. 1,a), в которой болометр, состоящий из высокоомного термочувствительного слоя $d$ и тонкого металлического 
поглотителя $a$, изготовлен на подложке $w 1$, а излучение падает через окно $w 2$, расположенное на значительном расстоянии (зазор g2) от болометра. Величина зазора $g 1$ (между болометром и подложкой) задается толщиной жертвенного слоя, который удаляется в конце процесса изготовления болометра и обычно составляет $2-3 \mu \mathrm{m}$. Между металлическим поглотителем и термочувствительным слоем находится тонкий изолирующий слой, который можно не учитывать, если его толщина достаточно мала, или включить в состав термочувствительного слоя, если он слабо от него отличается по величине коэффициента преломления. Под болометром на подложке $w 1$ может находиться отражатель $r$. В инвертированной конфигурации (рис. $1, b)$ излучение падает на болометр со стороны подложки $w 2$, на которой он изготовлен, а отражатель $r$ может находиться на выходном окне $w 1$. На окне $w 2$ (рис. $1, a$ ) или подложке $w 2$ (рис. $1, b)$, выполняющей роль входного окна, может быть нанесено антиотражающее покрытие $A R$.

Одно из основных различий этих двух конфигураций можно проиллюстрировать с помощью упрощенной модели. Пусть тонкий металлический поглотитель, толщина которого много меньше длины экранирования, расположен между двумя прозрачными средами (учитывая обозначения рис. 1, обозначим их как $w$ и $g$ ), имеющими разные диэлектрические проницаемости $\varepsilon_{w}$ и $\varepsilon_{g}$, а излучение падает со стороны среды $w$. Тогда коэффициенты пропускания $T$, отражения $R$ и поглощения $A$ падающей на границу раздела электромагнитной TЕ-волны, полученные в рамках так называемого инфракрасного приближения, будут заданы следующими соотношениями $[8,9]$ :

$$
\begin{gathered}
T=\frac{4 p_{w} p_{g}}{\left(p_{w}+p_{g}+f_{a}\right)^{2}}, \quad R=\frac{\left(p_{w}-p_{g}-f_{a}\right)^{2}}{\left(p_{w}+p_{g}+f_{a}\right)^{2}}, \\
A=\frac{4 p_{w} f_{a}}{\left(p_{w}+p_{g}+f_{a}\right)^{2}} .
\end{gathered}
$$

Здесь $p_{n}=\left(\varepsilon_{n} \varepsilon_{0} / \mu_{n} \mu_{0}\right)^{1 / 2} \cos \theta_{n}$ - адмиттанс наклонно распространяющейся ТЕ-волны в среде $n(n=w, g)$; $\varepsilon_{0}, \mu_{0}$ и $\varepsilon_{n}, \mu_{n}-$ абсолютные и относительные диэлектрические и магнитные проницаемости вакуума и среды $n$ соответственно; $f_{a}-$ слоевая проводимость (адмиттанс) тонкого металлического поглотителя; $\theta_{n}-$ угол, задающий направление распространения волны в слое $n$, который как обычно находится по закону преломления Снеллиуса. Все величины, входящие в (1), считаем действительными. Для ТМ-волны в соотношениях (1) необходимо заменить $p_{n}$ на $p_{n}^{\prime}=\left(\varepsilon_{n} \varepsilon_{0} / \mu_{n} \mu_{0}\right)^{1 / 2} / \cos \theta_{n}$, а при падении излучение со стороны среды $g$ переставить индексы $w$ и $g$.

Из соотношений (1) следует, что коэффициент пропускания не зависит от направления распространения излучения, а коэффициенты поглощения и отражения зависят. Поглощение достигает своего максимального значения $A=p_{w} /\left(p_{w}+p_{g}\right)$ при $f_{a}=p_{w}+p_{g}$, и в случае падения излучения со стороны среды с большим

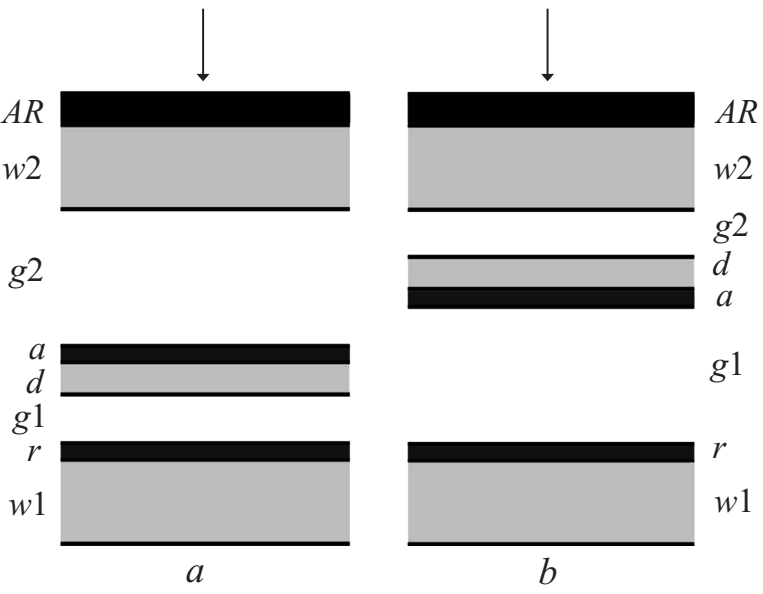

Рис. 1. Болометрические приемники традиционного $(a)$ и инвертированного $(b)$ типа.

адмиттансом оно оказывается больше, чем при падении со стороны среды с меньшим адмиттансом. При равных адмиттансах $p_{w}$ и $p_{g}$, также как и при нормальном падении инфракрасного излучения на расположенный в вакууме тонкий металлический поглотитель со слоевым сопротивлением $R_{a}=1 / f_{a}=\left(\mu_{0} / \varepsilon_{0}\right)^{1 / 2} / 2 \approx 188 \Omega / \square$, коэффициент поглощения равен 0.5 [9].

Такую предельно упрощенную модель можно применить к рассматриваемым нами болометрам в случае отсутствия входного окна $w 2$ (или его хорошего просветления с обеих сторон) и отражателя $r$, а также при отсутствии отражения от нижней границы подложки $w 1$, например, при хорошем ее просветлении (рис. 1,a). В случае инвертированного болометра (рис. $1, b)$ для этого потребуется отсутствие выходного окна $w 1$ (или его хорошее просветление с обеих сторон). Кроме того, так как обычно высота $h$ подвески микроболометров над подложкой составляет $2-3 \mu \mathrm{m}$, а толщина термочувствительного слоя $d$ менее $1 \mu \mathrm{m}$, то на интересующих нас длинах волн порядка $100 \mu \mathrm{m}$ этими толщинами можно пренебречь, и считать, что тонкий металлический поглотитель расположен на границе двух сред (между подложкой $w$ и вакуумом $g$ ).

В результате можно ожидать, что при отсутствии дополнительных резонаторов инвертированная болометрическая структура (рис. $1, b$ ), в которой излучение падает со стороны подложки, обладающей заметно большей диэлектрической проницаемостью по сравнению с вакуумом, должна обладать большим коэффициентом поглощения терагерцового излучения, чем традиционная структура (рис. 1,a). При этом, учитывая отсутствие частотной зависимости в (1), поглощение на низких частотах должно быть практически постоянным.

Основные особенности поведения коэффициента поглощения в болометрах традиционного типа в зависимости от частоты излучения, толщин зазоров $g_{1}, g_{2}$ и сопротивления металлического поглотителя представлены в [6,7]. С целью описания особенностей поглощения 
в болометрах инвертированного типа с дополнительным резонатором $g 1$, являющихся основным объектом исследования в настоящей работе, приведем аналитическое соотношение, применимое при следующих упрощениях. Как и ранее, пренебрежем толщиной зазора $g 2$ между болометром и подложкой $w 2$, на которой он изготовлен, и толщиной высокоомного термочувствительного слоя $d$, и будем считать, что тонкий металлический поглотитель болометра $a$ расположен на границе подложки $w 2$. Кроме того, будем полагать, что просветляющее покрытие $A R$ и отражатель $r$, показанные на рис. $1, b$, идеальны. Первое предположение позволяет считать, что электромагнитная волна, распространяющаяся в полупространстве, обладающем свойствами материала подложки $w 2$ (среда $w$ ), падает на структуру, состоящую из тонкого металлического поглотителя $a$, диэлектрического (в частности вакуумного) зазора $g 1$ (среда $g$ ) и отражателя $r$. Второе позволяет считать, что поглощение в отражателе не происходит. Тогда, используя соотношение (27) из работы [8] и устремив в нем слоевую проводимость $f_{r}$ отражателя $r$ к бесконечности, найдем коэффициент поглощения электромагнитной ТЕ-волны в рассматриваемом здесь упрощенном болометре инвертированного типа

$$
A=\frac{4 p_{w} f_{a}}{\left(p_{w}+f_{a}\right)^{2}+p_{g}^{2} \operatorname{ctg}^{2}\left(\kappa_{g} d_{g} \cos \theta_{g}\right)} .
$$

Это же соотношение можно получить, используя выражение для коэффициента отражения $R$ отражательного фильтра, состоящего из тонкого металлического слоя, отделенного от идеально отражающей подложки не поглощающим диэлектрическим слоем [9], и учитывая, что для такого фильтра $A=1-R$. Здесь $\kappa_{g}=(2 \pi / \lambda)\left(\varepsilon_{g} \mu_{g}\right)^{1 / 2} ; \lambda$ - длина волны излучения в вакууме; $d_{g}$ - толщина зазора $g 1$. Все величины, входящие в (2), считаем действительными. Так же как и ранее, в случае ТМ-волны в выражении (2) необходимо заменить $p_{n}$ на $p_{n}^{\prime}=\left(\varepsilon_{n} \varepsilon_{0} / \mu_{n} \mu_{0}\right)^{1 / 2} / \cos \theta_{n}$. Заметим, что соотношение (2) справедливо и для болометров традиционного типа, когда входное окно $w 2$ хорошо просветлено с обеих сторон (так, что дополнительный оптический резонатор, образованный зазором $g 2$, отсутствует). В этом случае можно считать, что излучение падает на болометр из вакуума и, следовательно, в соотношении (2) необходимо положить $p_{w}=p_{g}$. Здесь и далее будем считать зазоры $g 1$ и $g 2$ вакуумными. Следует особо отметить, что соотношение (2) нельзя применить даже для приблизительного анализа традиционного болометра с дополнительным резонатором $g$, поскольку последний находится по другую сторону от болометра по сравнению с тем, как это было предусмотрено при выводе соотношения (2); а при наличии отражателя $r$ важными являются оба зазора $g 1$ и $g 2$.

Из соотношения (2) следует, что поглощение нормально падающего $\left(\theta_{n}=0\right)$ излучения с длиной волны $\lambda$ окажется равным единице, если толщина $d_{g}=\lambda / 4$ и $f_{a}=p_{w}$. При длинах волн $\lambda \approx 100 \mu \mathrm{m}$ для болометров традиционного типа условие $d_{g}=\lambda / 4 \approx 25 \mu \mathrm{m}$ трудно реализовать, так как в хорошо отработанной технологии высота $h$ подвески болометров составляет $2-3 \mu \mathrm{m}$. В болометрах инвертированного типа такие большие величины зазора $g 1$ легко могут быть достигнуты путем установки пластины $w 1$ с отражателем $r$ на нужном расстоянии от болометра. Кроме того, из соотношения (2) следует, что в болометрах инвертированного типа ширина полосы поглощения, края которой определены, например, условием $A=0.5$, оказывается больше, чем в болометрах традиционного типа. Действительно, для болометров традиционного типа (в случае отсутствия дополнительного резонатора $g$ 2) $f_{a}=p_{w}=p_{g}=1 / 377 \mathrm{~S}$ и ширина полосы поглощения определяется условием $-2<\operatorname{ctg}\left(\kappa_{g} d_{g}\right)<2$. В болометрах инвертированного типа излучение падает на болометр со стороны подложки $w 2$, выполненной, например, из высокоомного кремния, имеющего показатель преломления $n$ в терагерцовой области, равный 3.4, так что $f_{a}=p_{w}=3.4 p_{g}$ и ширина полосы поглощения определяется условием $-6.8<\operatorname{ctg}\left(\kappa_{g} d_{g}\right)<6.8$. В частности, при $d_{g}=15 \mu \mathrm{m}$ максимум поглощения будет наблюдаться на длине волны $\lambda=60 \mu \mathrm{m}$, а условие $A>0.5$ будет выполняться в интервале 35-203 и 31-645 $\mu$ д для болометра традиционного и инвертированного типа соответственно, т.е. ширины полос поглощения отличаются в три раза. При заполнении большей части зазора $g 1$ нитридом кремния $(n \approx 2)$, как это сделано в [10] с целью решения технологических проблем осуществления высокой подвески болометров, полоса поглощения в болометре традиционного типа уменьшается до интервала 40-120 $\mu \mathrm{m}$.

В разд. 2 проведем численный расчет коэффициентов поглощения терагерцового излучения в болометрических структурах обоих типов, позволяющий учесть два зазора $g 1, g 2$, толщину термочувствительного слоя $d$, просветляющее покрытие и слоевые сопротивления поглотителя $a$ и отражателя $r$, что позволит провести сравнение реальных болометрических структур.

\section{2. Анализ коэффициентов поглощения в болометрических приемниках традиционного и инвертированного типа, проведенный в рамках матричного метода расчета}

Будем использовать известный матричный метод, развитый для расчета многослойных структур [11], детали которого применительно к болометрическим структурам, состоящим из произвольного количества слоев, включающих тонкий металлический поглотитель и отражатель, изложены в работе [8]. Под многослойной структурой, расположенной между двумя полупространствами с диэлектрическими проницаемостями $\varepsilon_{v}=\varepsilon_{s}=1$, будем понимать весь приемник, включая окно и подложку, выполненные из оптического кремния толщиной 


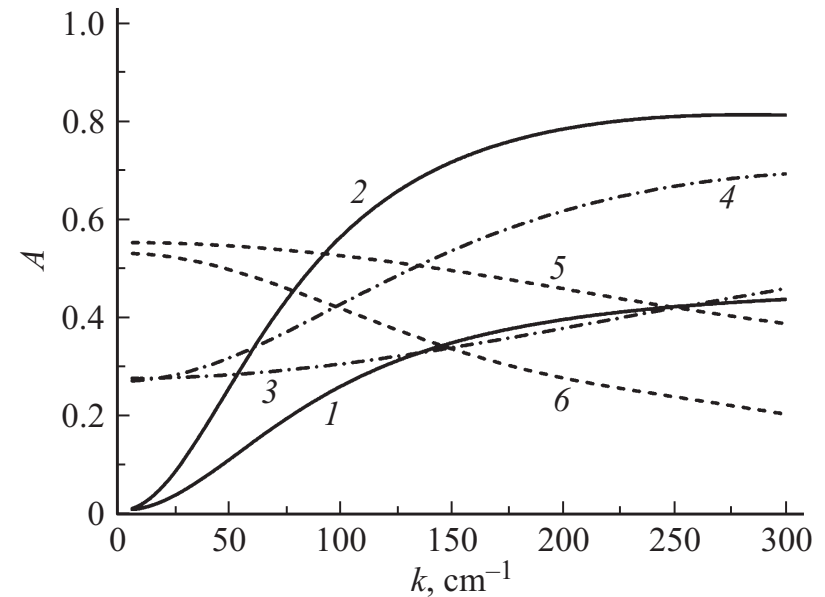

Рис. 2. Спектральные зависимости коэффициента поглощения в болометрах традиционного (1-4) и инвертированного $(5,6)$ типа при разных значениях $h$ и $R_{a}$ соответственно: $1-2.0$ и $57,2-5.7$ и $149,3-2.0$ и $128,4-5.7$ и $160,5-2.0$ и $83,6-5.7 \mu \mathrm{m}$ и $119 \Omega / \square$. При наличии отражателя $(1,2)$ и без него (3-6).

$700 \mu \mathrm{m}$. Рассчитанные коэффициенты поглощения усредним по полосе частот порядка $5 \mathrm{~cm}^{-1}$, чтобы устранить осцилляции коэффициентов поглощения, обусловленные интерференцией на окне и подложке, показатели преломления которых, так же как и высокоомного термочувствительного слоя, выполненного, например, из аморфного кремния толщиной $d_{d}=0.3 \mu \mathrm{m}$, приняты равными 3.4. Будем считать, что поглощение излучения происходит только в металлическом поглотителе $a$ и отражателе $r$. При этом нас будет интересовать только коэффициент поглощения $A$ в подвешенном над подложкой болометре, поскольку поглощение излучения именно в нем приводит к нагреву термочувствительного слоя и появлению сигнала болометра. Расчет проведен для случая падения излучения под прямым углом к поверхности болометра и применим к болометрам с латеральными размерами больше или порядка длины волны, так как не учитывались краевые эффекты. Удельная проводимость металлического поглотителя $\sigma_{a}$, ориентируясь на нихром, принималась равной $1 \cdot 10^{6} \mathrm{~S} / \mathrm{m}$. Слоевое сопротивление отражателя $R_{r}$ (при его наличии) выбиралось равным $0.4 \Omega / \square$, а его удельная проводимость $\sigma_{r}$, ориентируясь на золото и алюминий и учитывая ее понижение при малых толщинах слоев [12], принималась равной $2.5 \cdot 10^{7} \mathrm{~S} / \mathrm{m}$. Величину времени релаксации электронов в металлических слоях считали пренебрежимо малой по сравнению с периодом излучения в интересующем нас диапазоне частот.

Вначале рассмотрим упрощенный случай, когда имеется только непросветленная пластина кремния, на которой изготовлен болометр, а окно отсутствует, либо хорошо просветлено, так что его влиянием можно пренебречь. Приведенные на рис. 2 зависимости коэффициента поглощения $A$ от волнового числа $k=1 / \lambda$ рассчитаны при слоевых сопротивлениях поглотителя $R_{a}$, соответствующих максимальному поглощению на длине волны $\lambda=100 \mu \mathrm{m}$ для данной высоты подвески болометра $h$. В традиционной конфигурации (рис. $1, a)$, независимо от наличия или отсутствия отражателя, коэффициент поглощения в рассматриваемом диапазоне длин волн, в соответствии с [7], возрастает с ростом высоты подвески, а в инвертированной - понижается. Это дает преимущество для инвертированной конфигурации, так как увеличение высоты подвески связано с дополнительными технологическими трудностями. Отметим, что во всех рассмотренных на рис. 2 случаях для достижения максимального поглощения бо́льшая высота подвески болометра требует и большего сопротивления поглотителя, которое, однако, остается значительно меньше $377 \Omega / \square$, необходимых для достижения практически полного поглощения при наличии отражателя и при высоте подвески, равной четверти длины волны излучения. Из рис. 2 видно, что в соответствии с результатами проведенного предварительного анализа на низких частотах максимальное поглощение реализуется в случае инвертированной конфигурации болометра (рис. $1, b)$ и оно слабо зависит от частоты. В традиционной конфигурации при наличии отражателя коэффициент поглощения по мере понижения волнового числа $k$ квадратичным образом стремится к нулю. Это обусловлено тем, что на отражателе образуется узел электромагнитной волны и, следовательно, электрическое поле в области поглотителя, расположенного на малом (по сравнению с $\lambda$ ) расстоянии $h$ от отражателя, оказывается пропорциональным $k h$, что приводит к частотной зависимости коэффициента поглощения, пропорциональной $k^{2}$.

Известно, что в рамках традиционной конфигурации болометра коэффициент поглощения на заданной длине

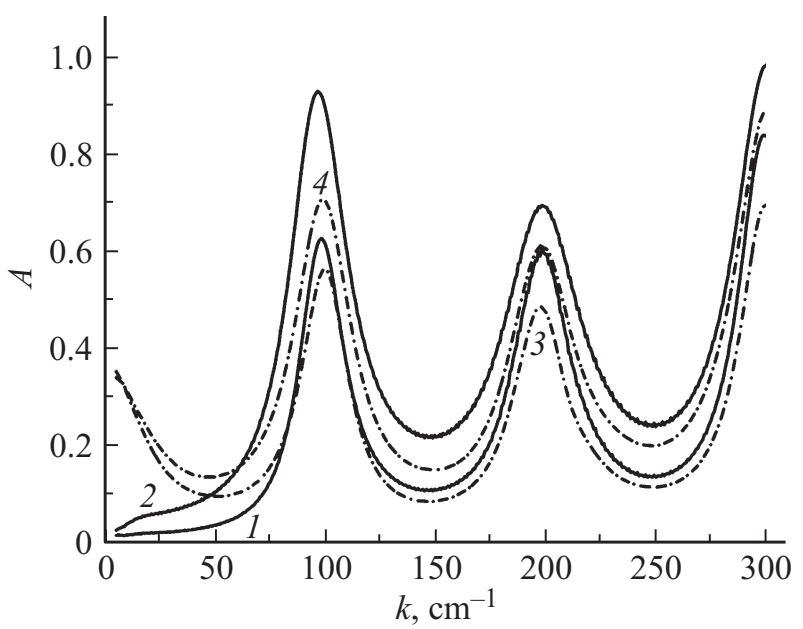

Рис. 3. Спектральные зависимости коэффициента поглощения в болометре традиционного типа с входным окном, расположенным на расстоянии $50 \mu \mathrm{m}$ от болометра при двух значениях $h$ и $R_{a}$ соответственно: 2.0 и $52(1,3), 5.7 \mu$ и и $93 \Omega / \square(2,4)$. При наличии отражателя $(1,2)$ и без него $(3,4)$. 


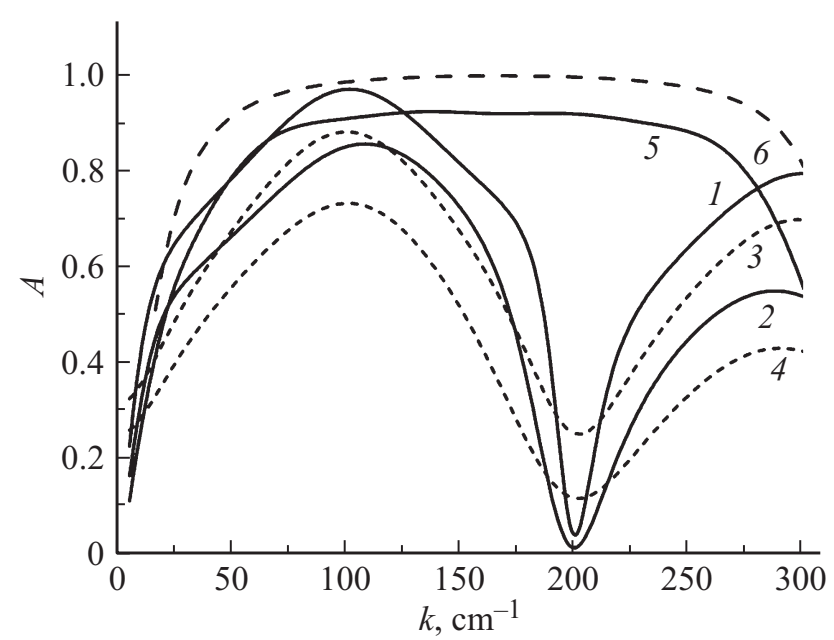

Pис. 4. Спектральные зависимости коэффициента поглощения в болометре инвертированного типа с выходным окном, расположенным на расстоянии $25(1-4)$ и $15 \mu \mathrm{m}(5,6)$ от болометра при двух значениях $h$ и $R_{a}$ соответственно: 2.0 и $126(1,3,5,6)$, $5.7 \mu \mathrm{m}$ и $198 \Omega / \square(2,4)$. При наличии отражателя $(1,2,5,6)$ и без него $(3,4)$.

волны можно повысить, установив просветленное с внешней стороны входное окно, так чтобы зазор $g 2$ между болометром и окном (рис. 1,a) был кратен половине данной длины волны [7]. На рис. 3 приведены спектральные зависимости коэффициента поглощения, рассчитанные при величине зазора, равном $50 \mu \mathrm{m}$. Здесь и на рис. 4 значения слоевых сопротивлений поглотителя $R_{a}$ выбирались по зависимостям 1 и 2 , соответствующим наличию отражателя, так чтобы коэффициент поглощения на длине волны $\lambda=100 \mu \mathrm{m}$ был максимален для данной высоты подвески болометра $h$. Просветляющее покрытие моделировалось одним слоем с показателем преломления $n=(3.4)^{1 / 2}$ и толщиной $d=25 / n \mu \mathrm{m}$. Видно, что наличие дополнительного резонатора между болометром и окном приводит к повышению коэффициента поглощения вблизи $k \approx 100 \mathrm{~cm}^{-1}$ почти до единицы, но при этом поглощение становится сильно селективным, что не всегда желательно, например, при необходимости детектирования широкополосного излучения.

Применение дополнительного резонатора между подложкой и выходным окном приводит к повышению коэффициента поглощения и в случае инвертированной конфигурации болометра (рис. 4). В отличие от традиционной конфигурации здесь оптимальным для получения максимального поглощения на длине волны $100 \mu \mathrm{m}$ является зазор $g$, равный $25 \mu \mathrm{m}$. При наличии отражателя на выходном окне и однослойного просветляющего покрытия, аналогичного используемому выше, здесь также удается получить коэффициенты поглощения близкие к единице. Кроме того, поглощение является более широкополосным, чем в болометрическом приемнике традиционного типа, а отсутствие отражателя меньше понижает поглощение. Уменьшение величины зазора $g 1$ до $15 \mu \mathrm{m}$ и применение четырехслойного просветляющего покрытия на внешней стороне подложки $w 2$ позволяет получить эффективное поглощение $(A>0.8)$ в широкой полосе $50-270 \mathrm{~cm}^{-1}$ (кривая 5 на рис. 4). Диэлектрические проницаемости $\varepsilon_{n}$ просветляющих слоев были равны $\varepsilon_{n}=7.4 ; 3.5 ; 1.8$ и 1.3 , уменьшаясь с удалением от подложки $w 2$, а их толщины были заданы соотношением $15 /\left(\varepsilon_{n}\right)^{1 / 2} \mu$ m. Кривая 6 на рис. 4, рассчитанная по соотношению (2) при тех же параметрах, что и кривая 5 , показывает удовлетворительную применимость данного соотношения. Пониженные величины поглощения на кривой 5 в основном обусловлены учетом конечного значения $h$ и ухудшением качества просветляющего покрытия на краях полосы просветления.

\section{3. Возможность использования болометров инвертированного типа с целью создания широкополосных матричных приемников терагерцового диапазона}

Матричные микроболометрические приемники (ММБП) традиционной конфигурации изготавливаются на кремниевых подложках, в которых предварительно выполнены схемы считывания сигналов (мультиплексоры). Такие подложки обычно легированы и не прозрачны в терагерцовом диапазоне. Кроме того, под болометрами располагаются элементы схемы считывания, такие как транзисторные ключи и шины, что также не позволяет выполнять засветку болометра со стороны подложки. Практическая реализация широкополосных ММБП инвертированного типа для терагерцового диапазона длин волн может быть выполнена при использовании нелегированного, оптического кремния в качестве подложки, тыльная сторона которой после изготовления мультиплексора должна быть отполирована. Элементы мультиплексора, в том числе и локальные области легирования, должны быть вынесены из-под микроболометров в межпиксельные зазоры, которые в случае изготовления болометров с латеральными размерами $\sim 100 \mu \mathrm{m}$ (сравнимыми с длиной волны излучения) могут быть достаточно широкими. С учетом возможности засветки микроболометров со стороны подложки кремниевый кристалл с ММБП плотно, например с помощью тонкого прозрачного в терагерцовом диапазоне клея, устанавливается на входное кремниевое окно вакуумного корпуса, просветленное снаружи в требуемом диапазоне длин волн. После чего на необходимом от ММБП расстоянии устанавливается выходное окно, в общем случае с металлическим отражателем, нанесенным на его внутреннюю сторону. Отметим, что использование болометрических приемников инвертированного типа затрудняет применение термоэлектрических охладителей для стабилизации температуры ММБП, что не является существенным препятствием в силу тенденции все 
более широкого применения TEC-less технологии [13], обеспечивающей работу ММБП без стабилизации его температуры.

\section{Заключение}

В настоящей работе предложен инвертированный тип конструкции болометрического приемника для терагерцового спектрального диапазона, в котором в отличие от традиционного типа излучение падает не со стороны прилегающего к болометру вакуума, а со стороны подложки, на которой он изготовлен. На основе проведенного расчета коэффициентов поглощения терагерцового излучения в болометрических структурах с тонким металлическим поглотителем показано, что в области низких частот инвертированный тип обеспечивает большее поглощение, чем традиционный. Применение дополнительного резонатора в виде зазора между болометром и входным или выходным окном позволяет получать коэффициенты поглощения близкие к единице, при этом болометры с традиционной конфигурацией становятся узкополосными, а с инвертированной - остаются широкополосными. В частности, инвертированный тип болометра может обеспечить высокоэффективное поглощение излучения в диапазоне от 50 до $270 \mathrm{~cm}^{-1}$. На основе болометров инвертированного типа предложен вариант конструкции высокоэффективного широкополосного матричного микроболометрического приемника терагерцового диапазона.

\section{Список литературы}

[1] Terahertz Spectroscopy: Principles and applications / Ed. S.L. Dexheimer. Publisher: CRC Press, 2008. 331 p.

[2] Chan W.L., Deibel J., Mittleman D.M. // Rep. Prog. Phys. 2007. Vol. 70. N 8. P. 1325-1379.

[3] Lee A.W.M., Hu Q. // Opt. Lett. 2005. Vol. 30. N 19. P. 2563 2565.

[4] Lee A.W.M., Williams B.S., Kumar S., Hu Q., Reno J.L. // IEEE Photon. Technol. Lett. 2006. Vol. 18. N 13. P. 14151417.

[5] Dem'yanenko M.A, Esaev D.G., Knyazev B.A., Kulipanov G.N., Vinokurov N.A. // Appl. Phys. Lett. 2008. Vol. 92. P. 131116

[6] Oda N., Yoneyama H., Sasaki T., Sano M., Kurashina S., Hosako I., Sekine N., Sudoh T., Irie T. // Proc. SPIE. 2008. Vol. 6940. P. 69402Y.

[7] Oda N., Sano M., Sonoda K., Yoneyama H., Kurashina S., Miyoshi M., Sasaki T., Hosako I., Sekine N., Sudou T., Ohkubo S. // Proc. SPIE. 2011. Vol. 8012. P. 80121B.

[8] Демьяненко М.А. // Оптический журн. 2017. Т. 84. Вып. 1. C. $48-57$.

[9] Hadley L.N., Dennison D.M. // J. Opt. Soc. Am. 1947. Vol. 37. N 6. P. 451-465.

[10] Nemoto N., Kanda N., Imai R., Konishi K., Miyoshi M, Kurashina S., Sasaki T., Oda N., Kuwata-Gonokami M. // IEEE Trans. on Terahertz Sci. Technol. 2016. Vol. 6. N 2. P. $175-182$.
[11] Борн М., Воль $\varnothing$ Э. Основы оптики. М.: Наука, 1973. 720 с.

[12] Camacho M., Oliva A.I. // Microelectronics J. 2005. Vol. 36. N 3-6. P. 555-558.

[13] Howard P.E., Clarke J.E., Li C., Yang J.W., Wong W.Y., Bogosyan A. // Proc. SPIE. 2003. Vol. 5074. P. 527-536. 